\title{
Motivos de manutenção e abandono no voleibol universitário
}

\section{Reasons for dropout and maintenance of sports career college volleyball athletes}

\author{
Marco Túlio Silva Batista ${ }^{1, *}$ \\ Leandro Rafael Leite ${ }^{2}$ \\ Mariane Faria Braga Bacelar ${ }^{3}$ \\ Marcelo de Castro Teixeira ${ }^{4}$ \\ Alessandro Teodoro Bruzi ${ }^{4}$
}

\section{Resumo:}

Objetivo: Analisar os motivosque influenciam as fases de uma carreira esportiva em função do sexo, idade e tempo de prática (TP).Métodos: A amostra foi composta por atletas de Voleibol, universitários, de ambos os sexos. Foram utilizadas as subescalas de Manutenção e Abandono do Questionário MIMCA-BR. Resultados: Os atletas continuaram a praticar Voleibol na universidadedevido à sua capacidade física $(U=0,623 ; p=0,027)$; provavelmente abandonariam a prática por não concretizarem de sonhos $(U=0,713 ; p=0,019)$. Já atletas do sexo feminino abandonariam por falta de apoio familia $r(U=932 ; p=0,001)$. Observou-se também diversos motivos que se alteraram em relação à idade e ao TP. Conclusão: os motivos se alteram em função do sexo, idade e TP.

Palavras-chave: esporte, universidade, motivação.

\section{Abstract:}

Objective: Analyze the reasons that influence the sport career as a function of sex, age and time of practice (TP). Methods: The sample consisted of college Volleyball athletes from both genders. The maintenance and abandonment sub scales of the MIMCA-BR Questionnaire were used. Results: Male athletes continued to practice volleyball at university because of their physical ability $(U=0.623 ; p=$ $0.027)$; they would probably abandon the practice because they did not dream $(U=0.713, p=0.019)$. On the other hand, the female athletes would abandon due to lack of family support $(U=932, p=$ $0.001)$. There were also several reasons that changed in relation to age and PT. Conclusion: the motives change according to gender, age and PT.

Keywords: sport, university, motivation.
Afiliação dos autores

${ }^{1}$ Universidade Federal de Minas Gerais, Belo Horizonte, Minas Gerais. Brasil.

${ }^{2}$ Centro Mineiro de Ensino Superior, Campo Belo, Minas Gerais. Brasil.

${ }^{3}$ Auburn University, Auburn,

Alabama. Estados Unidos da

América.

${ }^{4}$ Universidade Federal de Lavras, Lavras, Minas Gerais. Brasil.

*Autor correspondente

Rua Flor do Baile, 141. Jardim Montanhês. Belo Horizonte, Minas Gerais, Brasil.

e-mail:

marco-tulio1992@hotmail.com

Conflito de interesses

Os autores declararam não haver conflito de interesses.

Processo de arbitragem

Recebido: 08/05/2018 Aprovado: 22/07/2018 


\section{Introdução}

Discussões queproblematizam periodizações de treinamentos, especialização precoce em e questões psicológicas aplicadas ao esporte estão em evidência. No entanto, nota-se a necessidade de apresentar a discussão acerca da conciliação entre as formaçõesesportiva e escolar para jovens atletas envolvidos nessa "dupla carreira"1.

Práticas esportivas expõem diferentes características nos processos educacionais e nas mudanças físicas e desenvolvimentistas durante a infância e adolescência $^{2} e$, podem ser influenciadas por diversos fatores, como fatores sociais, físicos, táticos, técnicos e psicológicos ${ }^{3-6}$. Ao pensar no contexto psicossocial, a motivação aparece como uma das principais explicações para elucidar as decisões do atleta no decorrer da carreira esportiva, como o aumento da motivação devido à percepção de competência estaria relacionada à continuação da prática esportiva ${ }^{4,6}$. Todavia, uma desmotivaçãopode afetar a meta a ser atingida nesse processo e culminar noabandono do esporte 6 .

Nas últimas décadas, as investigações têm questionado acerca da motivação para continuar a praticaresporte e o que influencia na decisão de abandonar essa prática ${ }^{4,7,8}$. Alguns dos motivos relacionados à manutenção da prática estão atreladas à vontade dos pais e/ou condicionadas à participação de amigos ${ }^{3,9}$ e também à melhora técnica na modalidade ${ }^{2}$.O abandono tem sidoatreladoà dificuldade de relacionar estudos com exigências de treinamentos, à desmotivação com 0 exporte e à especialização precoce ${ }^{10,11}, 0$ avanço da idade e 0 estresse devido ao Tempo de Prática (TP) ${ }^{12}$, além de lesões ${ }^{13}$.

Já o abandono da prática esportiva é comum quando o atleta alcança determinada idade na transição para a vida adulta. Simultaneamente acontece um evento psicossocial de extrema importância: a inserção no ensino superior. Esse se caracteriza como um dos fatores de interferência na carreira esportiva e, portanto, merece consideração nessa linha de estudos. Nesse momento de transição, os desportistas se deparam com uma situação binária: continuar a carreira esportiva, visando o profissionalismo, ou ingressar no ensino superior e estabelecer uma carreira fora do esporte? Na cultura esportiva brasileira, essa decisão pressupõe em optar por um dos dois caminhos, pois o esporte universitário não tem influência ou representa muito pouco no processo continuo de formação de atletas ${ }^{14}$. A inserção do jovem na universidade é um período considerado crítico para a manutenção da prática esportiva ${ }^{15}$. A tendência, nessa situação, é de que o universitário abandone o esporte em função de uma maior dedicação ao estudo e/ou trabalho ${ }^{16}$.

No entanto, mesmo que em minoria, algumas equipes profissionais são financiadas por Instituição de Ensino Superior (IES) privada, nas quais os atletas recebem salários para competir por essas equipes em competições federadas e, em contrapartida, representam as IES privada em competições universitárias, em âmbito estadual e nacional. Algumas IES públicastêm proporcionado ao estudante a possibilidade de continuar a prática esportiva, seja visando a manutenção da saúde ou até mesmo a participação em equipes para a disputa de competições universitárias. Em função dessa oportunidade, levanta-se a seguinte questão: será que para universitários do ensino público os motivos de manutenção e abandono são os mesmospropostos pelos estudos que investigaram tais motivos com não universitários?

Assim, o presente estudo teve como objetivo analisar dentro da carreira esportiva universitária de voleibolistas os diferentes motivos de manutenção da prática após ingressar no ensino superior e possíveis motivos de abandono em cada sexo, além da relação com a idade e o TP.

\section{Métodos}

\section{Participantes}

Esse estudo contou com a participação de 143 atletas de Voleibol, estudantes de universidades pública, sendo 61 do sexo masculino (idade: $21,72 \pm 2,46$ anos; TP: $9,28 \pm 4,49$ anos) e 82 do sexo feminino (idade: $21,57 \pm 3,44$ anos; TP: $9,56 \pm 5,24$ anos), envolvidos em competições universitárias no Estado de Minas Gerais no ano de 2015.

\section{Instrumentos}

Utilizou-se das subescalas de Manutenção e Abandono presentes no Questionário de Motivos de Início, Manutenção, Mudança e Abandono do Esporte (MIMCA-BR). Tal instrumento foi desenvolvido na língua espanhola ${ }^{17} \mathrm{com}$ o nome Início, Manteniemento, Cambio e Abandono, sendo posteriormente traduzido e validado para a Língua Portuguesa ${ }^{7}$.

As subescalas de interesse contêm 27 questões: Manutenção (17) e Abandono (10). As respostas baseiam-se em uma escala tipo Likert de 5 pontos sendo (1) "total desacordo", (2) "pouco desacordo", (3) "de acordo", (4) "muito de acordo" e (5) "totalmente de acordo". Esse instrumento tem mostrado sua consistência interna em diversos estudos oscilando o alfa de Cronbach entre 0,82 e $0,94^{18}$. Valores de alfa indicando consistência interna foram identificados para esse estudo: 0,832 Manutenção e 0,847 Abandono.

\section{Procedimentos}

O questionário foi aplicado pelos próprios pesquisadores durante competições universitárias organizadas pela Federação Universitária Mineira de Esportes (FUME), em local restrito. A FUME e os treinadores e/ou representantes de equipe autorizaram os pesquisadores a aplicarem o questionário. Dessa forma, após o consentimento formal dos participantes, os pesquisadores informaram a respeito do objetivo da pesquisa e, em seguida, entregaram o questionário para o preenchimento. $O$ preenchimento foi individual e teve duração aproximada de 10 minutos. Todos os dados obtidos foram arquivados em um computador de acesso restrito para garantir que nenhuma informação pessoal fosse indevidamente divulgada.

Os universitários foram divididos em três grupos no que se refere à idade: Grupo Idade 1 (GI-1) - universitários com idade entre 18 e 21 anos; Grupo Idade 2 (Gl-2) - idade entre 22 e 26 anos e Grupo Idade 3 (GI-3) - Idade entre 27 e 30 anos. Em relação ao TP, esses universitários foram redivididos em outros três grupos: o Grupo Tempo de Prática 1 (GTP-1) - TP entre 1 e 7 anos; Grupo Tempo de Prática 2 (GTP-2) - TP entre 8 e 14 anos e o Grupo Tempo de Prática 3 (GTP-3) - TP entre 15 e 20 anos.

\section{Considerações éticas}

O projeto foi aprovado pelo Comitê de Ética em Pesquisas com Seres Humanos da Universidade Federal de Lavras/Minas Gerais sob número CAAE: 41436214.8.0000.5148. Durante a coleta de dados foi solicitadoaos atletas a assinatura do Termo de Consentimento Livre e Esclarecido (TCLE), conforme resolução CNS no. 466/12 em concordância com a realização da investigação, o que possibilitou sua divulgação sem inconvenientes. Foi garantida aos atletas a confidência dos dados descritos e respondidos nos questionários. A realização do estudonão gerou riscos para os atletas.

\section{Análise estatística:}

As variáveis independentes do presente estudo foram sexo, Idade e TP, enquanto as variáveis dependentes foram os motivos de Manutenção e Abandono da prática do Voleibol.

Em uma etapa preliminar realizou-se uma análise para identificar a confiabilidade interna do questionário para essa amostra através do teste alfa de Cronbach. Conforme o teste de Kolmogorov-Smirnov, o conjunto de dados não atingiu os pressupostos para a realização de análises inferenciais paramétricas. Dessa forma,os motivos foram observados por meio de frequência de respostas e os motivos de cada subescala foram comparados entre os sexose a determinação do tamanho do efeito (F) por meio do teste $U$ de Mann-Whitney. O teste de Correlação de Spearman foi utilizado para verificar correlação entre os motivos de Manutenção e Abandono em função da Idade e do TP. Os dados foram analisados através do pacote estatístico SPSS versão 20.0 e foi mantido nível de significância de $p \leq 0,05$. Enquanto o poder estatístico (1- $\beta$ ) igual a 0,831 se deu através do software Gpower versão 3.1.9.2.

\section{Resultados}


Existe diferença entre os sexos com relação aos motivos considerados para manutenção e para o abandono?

Considerando os principais motivos para a manutenção no esporte, a comparação identificou que se manter no esporte para melhorarem fisicamente foi mais relevante para atletas do sexo masculino $(U=0,623 ; p=0,027)$. O mesmo ocorreu em

Tabela 1

Principais motivos de cada sexo para Manutenção e Abandono.

\begin{tabular}{|c|c|c|c|c|c|}
\hline Motivos & Sexo & Frequência (\%) & Média & $p$ & $\mathrm{~F}$ \\
\hline \multicolumn{6}{|c|}{ Manutenção } \\
\hline $\begin{array}{l}\text { Praticando esporte melhoro } \\
\text { fisicamente }\end{array}$ & $\begin{array}{l}\text { Masculino } \\
\text { Feminino }\end{array}$ & $\begin{array}{l}57,4 \\
52,5\end{array}$ & $\begin{array}{l}4,31( \pm 0,58) \\
3,24( \pm 1,30)\end{array}$ & 0,027 & 1,06 \\
\hline \multicolumn{6}{|c|}{ Abandono } \\
\hline $\begin{array}{l}\text { Se meus sonhos como atleta não se } \\
\text { cumprissem }\end{array}$ & $\begin{array}{c}\text { Masculino } \\
\text { Feminino }\end{array}$ & $\begin{array}{l}83,2 \\
44,1\end{array}$ & $\begin{array}{l}2,49( \pm 1,22) \\
1,96( \pm 0,69)\end{array}$ & 0,019 & 0,53 \\
\hline Se minha família não me apoiasse & $\begin{array}{l}\text { Masculino } \\
\text { Feminino }\end{array}$ & $\begin{array}{l}72,1 \\
78,8\end{array}$ & $\begin{array}{l}1,49( \pm 0,32) \\
3,89( \pm 0,80)\end{array}$ & 0,001 & 3,94 \\
\hline $\begin{array}{l}\text { Se meus pais não gostassem do meu } \\
\text { esporte }\end{array}$ & $\begin{array}{c}\text { Masculino } \\
\text { Feminino }\end{array}$ & $\begin{array}{l}77,1 \\
86,6 \\
\end{array}$ & $\begin{array}{l}1,93( \pm 0,92) \\
3,56( \pm 0,53)\end{array}$ & 0,006 & 2,17 \\
\hline
\end{tabular}

Existe relação entre a idade dos(as) atletas e os motivos considerados para manutenção e para o abandono?

No que se refere aos motivos relacionados à Idade, os resultados demonstram que, para as atletas mais velhas,a manutenção no esporte se mostrou associada aos motivos: se dar bem com o treinador, às melhorias na condição física e ao estar treinando, mesmo com clima adverso.A possibilidade de

relação ao abandono da modalidadese seus sonhos não se cumprissem $(U=0,713 ; p=0,019)$. Já as atletas do sexo indicaram considerar o abandono pela falta de apoio da família $(U=932 ; p=0,001)$ e o desgosto dos pais pelo esporte $(U=0,891$; $\mathrm{p}=0,006)$ (Tabela 1).

Tabela 2

Correlação da Idade com os motivos de Manutenção e Abandono.

\begin{tabular}{|c|c|c|c|c|c|}
\hline \multirow{2}{*}{ Motivos } & \multirow{2}{*}{ Grupo } & \multicolumn{2}{|c|}{ Masculino } & \multicolumn{2}{|c|}{ Feminino } \\
\hline & & $r$ & $p$ & $\mathrm{R}$ & $p$ \\
\hline \multicolumn{6}{|c|}{ Manutenção } \\
\hline \multirow{4}{*}{ Dou-me bem com meu treinador } & $\mathrm{Gl}-1$ & $-0,210$ & 0,360 & 0,116 & 0,536 \\
\hline & Gl-2 & 0,013 & 0,943 & $-0,072$ & 0,671 \\
\hline & GI-3 & $-0,258$ & 0,318 & 0,597 & $0,031^{*}$ \\
\hline & Gl-1 & 0,090 & 0,699 & 0,189 & 0,308 \\
\hline \multirow{3}{*}{ Praticando esporte melhoro fisicamente } & GI-2 & $-0,123$ & 0,502 & 0,616 & $0,039^{*}$ \\
\hline & GI-3 & 0,088 & 0,775 & 0,923 & $0,025^{*}$ \\
\hline & Gl-1 & $-0,246$ & 0,351 & 0,214 & 0,239 \\
\hline \multirow[t]{2}{*}{ Apesar do clima adverso, vou treinar } & GI-2 & $-0,266$ & 0,141 & $-0,205$ & 0,223 \\
\hline & GI-3 & $-0,209$ & 0,492 & 0,507 & $0,038^{*}$ \\
\hline \multicolumn{6}{|c|}{ Abandono } \\
\hline \multirow{3}{*}{ Se trabalho/estudo não permitisse render o máximo } & Gl-1 & 0,173 & 0,454 & $-0,182$ & 0,319 \\
\hline & Gl-2 & 0,113 & 0,546 & 0,180 & 0,287 \\
\hline & GI-3 & $-0,487$ & 0,092 & $-0,808$ & $0,024^{*}$ \\
\hline & Gl-1 & 0,157 & 0,516 & 0,047 & 0,797 \\
\hline \multirow[t]{3}{*}{ Não tivesse capacidade física para o meu esporte } & GI-2 & 0,063 & 0,732 & 0,286 & 0,086 \\
\hline & GI-3 & $-0,137$ & 0,655 & 0,913 & $0,007^{*}$ \\
\hline & Gl-1 & $-0,915$ & $0,025^{*}$ & $-0,824$ & $0,017^{*}$ \\
\hline \multirow[t]{2}{*}{ Se não me desse bem como com o treinador } & GI-2 & $-0,187$ & 0,305 & 0,097 & 0,567 \\
\hline & GI-3 & $-0,262$ & 0,387 & $-0,064$ & 0,818 \\
\hline
\end{tabular}

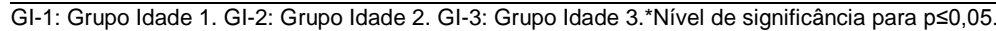

abandonar a modalidade se mostrou associada à dificuldade de rendimento máximo em função de estudo e trabalho e baixa condição física nas atletas mais velhas e ao fato de não estar se dando bem com o treinador nas atletas mais jovens. No sexo masculino, o fato de não se dar bem com o treinador se relacionou com a possibilidade de abandono para os atletas mais jovens (Tabela 2).
Existe relação entre o TP dos(as) atletas e os motivos considerados para manutenção e para o abandono?

Sobre os motivos considerados para a manutenção no esporte, a relação com a estética esteve associada negativamente a um maior TP em atletas do sexo feminino. Sobre a probabilidade de abandono, a dificuldade para render o máximo nos treinos e jogos se relacionou positivamente ao menor TP em atletas do sexo masculino. Já para o possível abandono deatletas de ambos os sexos, a incompatibilidade entre treino e trabalho esteve positivamente associado ao menor TP, enquanto adificuldade para se dar bem com o treinador e não conseguir os resultados desejados se relacionaram negativamente a um menor TP (Tabela 3).

\section{Discussão}

Ao longo das últimas décadas, investigações acerca dos possíveis motivos que levariam o atleta a abandonar a carreira esportiva têm sido alvo de questionamentos em vários estudos $^{4,7,8,19-24}$. Esses estudos têm mostrado que os atletas consideram vários motivos para o fim da carreira esportiva, como "não terem capacidades físicas para o esporte", Tabela 3

Correlação do TP com os motivos de Manutenção e Abandono. "desmotivação com a prática", "relação negativa com o treinador", "intensidade dos treinamentos/cansaço", "pressão dos pais", "lesões", "estresse","dificuldades, necessidade de outras atividades" e, principalmente, "impossibilidade de conciliar o esporte com estudo ou trabalho". O objetivo desse estudo foi o de analisar, de forma retrospectiva,os motivos que influenciaram determinados indivíduos à manutençãoda prática no Voleibol, bem como aqueles que poderiam influenciar a mudança e a o abandono dessa modalidade. Em função disso, os resultados dessa investigação foramdiscutidos observando as diferenças entre sexos e analisando as relações com a idade e com o TP.

A família foi outro fator apontado como influente, especialmente para o sexo feminino, no que diz respeito àmanutenção no Voleibol. Resultados semelhantes foram encontrados $^{25}$, o qual foi investigado atletas de diversas modalidades de ambos os sexos. Nesse caso, o apoio familiar para a prática dessa modalidade pode ser reflexo de uma velha discussão cultural acerca de como a questão de gênero é admitida em nossa sociedade. Assim, dentro dos padrões de gênero adotados, o Voleibol é considerado uma atividade feminina como cozinhar, ler poesia e cuidar de crianças ${ }^{26-28}$. 


\begin{tabular}{|c|c|c|c|c|c|}
\hline \multirow{2}{*}{ Motivos } & \multirow{2}{*}{ Grupo } & \multicolumn{2}{|c|}{ Masculino } & \multicolumn{2}{|c|}{ Feminino } \\
\hline & & $r$ & $p$ & $r$ & $p$ \\
\hline \multicolumn{6}{|c|}{ MANUTENÇÃO } \\
\hline \multirow{4}{*}{ Praticando esporte melhoro fisicamente } & GTP-1 & $-0,148$ & 0,522 & 0,331 & 0,686 \\
\hline & GTP2 & $-0,281$ & 0,122 & $-0,255$ & 0,117 \\
\hline & GTP3 & 0,197 & 0,519 & $-0,520$ & $0,042^{*}$ \\
\hline & GTP-1 & $-0,133$ & 0,555 & 0,397 & 0,160 \\
\hline \multirow[t]{3}{*}{ Com o esporte melhoro a minha imagem física } & GTP2 & $-0,174$ & 0,289 & 0,196 & 0,232 \\
\hline & GTP3 & $-0,075$ & 0,827 & $-0,448$ & $0,043^{*}$ \\
\hline & GTP-1 & 0,326 & 0,139 & 0,367 & 0,196 \\
\hline \multirow[t]{2}{*}{ O esporte me torna mais atraente } & GTP2 & 0,122 & 0,793 & $-0,160$ & 0,132 \\
\hline & GTP3 & $-0,381$ & 0,248 & $-0,700$ & $0,026^{*}$ \\
\hline \multicolumn{6}{|c|}{ ABANDONO } \\
\hline \multirow{4}{*}{ Treinar fosse incompatível com o meu Trabalho/Estudo } & GTP-1 & 0,599 & $0,026^{*}$ & 0,542 & $0,041^{*}$ \\
\hline & GTP2 & $-0,096$ & 0,626 & $-0,322$ & $0,046^{*}$ \\
\hline & GTP3 & $-0,427$ & 0,191 & $-0,316$ & 0,270 \\
\hline & GTP-1 & 0,631 & $0,020^{*}$ & 0,139 & 0,482 \\
\hline \multirow[t]{3}{*}{ Se trabalho/estudo não permitisse render o máximo } & GTP2 & 0,130 & 0,520 & 0,275 & 0,133 \\
\hline & GTP3 & $-0,499$ & 0,118 & $-0,196$ & 0,501 \\
\hline & GTP-1 & $-0,630$ & $0,021^{*}$ & $-0,296$ & $0,048^{*}$ \\
\hline \multirow[t]{3}{*}{ Se não me desse bem com o treinador } & GTP2 & $-0,021$ & 0,914 & 0,036 & 0,826 \\
\hline & GTP3 & $-0,203$ & 0,549 & $-0,075$ & 0,799 \\
\hline & GTP-1 & $-0,699$ & $0,012^{*}$ & $-0,818$ & $0,008^{*}$ \\
\hline \multirow[t]{2}{*}{ Se não conseguisse os resultados que desejo } & GTP2 & $-0,211$ & 0,280 & 0,084 & 0,610 \\
\hline & GTP3 & $-0,107$ & 0,753 & $-0,243$ & 0,403 \\
\hline
\end{tabular}

GTP-1: Grupo Tempo de Prática 1. GTP-2: Grupo Tempo de Prática 2. GTP-3: Grupo Tempo de Prática 3. *Nível de significância para p $\leq 0,05$.

Motivos relacionados às capacidades físicas e à estética também foram citados como fundamentais para a manutenção da prática e corroboram com os achados deMata ${ }^{29}$. Tais motivos podem ser explicados pelo processo que o indivíduo vivencia ao construir uma autoimagem corporal, experimentando novas sensações e reconhecendo seus limites ${ }^{29,30}$.

Em se tratando de abandono do esporte, o presente estudo apresentou diferenças entre os sexos.Afalta de apoio dos pais e o não alcancede sonhos se destacaram como possíveis motivos que causariam o abandono da modalidade pelo sexo feminino e masculino, respectivamente. Esses resultados se diferem daqueles apresentados, predominantemente, pela literatura, a saber, "prejuízo nos estudos", "intensidade dos treinamentos/cansaço", "pressão dos pais" e "dificuldades e necessidade de outras atividades" ${ }^{\prime 4,8,9,31,32}$. Essa diferença pode estar atrelada, principalmente, ao fato de os participantes desse estudo estarem experimentandoa carreira esportiva após superarem a incerteza gerada pelo processo de ingresso no ensino superior.

Os resultados da correlação entre os motivos paraManutenção eAbandonoea idade, indicam que quanto maior a idade, maiores os índices de evasão noesporte. Nota-se que, com o passar dos anos, os escores aumentam, evidenciando as dificuldades de conciliar a vida esportiva com os estudos. Estes resultados são coincidentescom os resultados obtidos por pesquisas realizadas nas Universidades de León e Múrcia sobre as razões para o abandono da prática esportiva entre os estudantes universitários ${ }^{33}$. $\mathrm{O}$ fator de desmotivação pode estar associadoao estresse no esporte e em um estudo com seleções femininas estaduais de Voleibol $^{12}$, com idades entre 15 e 18 anos, apresentam que o estresse no esporte está relacionado à idade e ao TP, sendo que atletas mais jovens e com menos TP estão mais expostos a essa situação.

As associações entre os motivos em cada fase da carreira esportiva e o TP, revelaram diferentes valores em função do sexo. Para os universitários, quanto maior o TP, maior a chance das atletas se manterem praticando por vaidade física; menor a chance de abandono por não se darem bem com o(a) treinador(a). Para estudar a carreira esportiva é preciso analisar esse processo com um olhar multifacetado, a partir do perfil do atleta, da cultura organizacional da modalidade esportiva e da condição socioeconômica do desportista ${ }^{5}$. Sob essa ótica, estudos de caráter multidisciplinar são fundamentais para auxiliarem no planejamento de programas de treinamento direcionados a um grupo específico, pois o conhecimento sobre esses elementos aumenta a possibilidade de permanência do indivíduo no esporte ${ }^{3}$. Dentro dessa multidisciplinaridade, estudos são realizados para explicar a carreira esportiva a partir dos aspectos físicos, táticos, técnicos e psicossociais ${ }^{3,4,6,9,34,35}$.

O diferencial do presente estudo, portanto, foi a verificação de que os principais motivos, possíveis causadores de evasão do Voleibol, estão relacionados, direta ou indiretamente, à Idade e ao TP. Nesse sentido ${ }^{14}$ pontuam que um motivo importante para 0 abandono precoce no esporte éo fato de o modelo universitário brasileiro não ter um papel de continuidade na formação de atletas. Isso faz com que o atleta que almeja ter uma carreira profissional além do esporte, tenha de decidir entre continuar praticando visando à possibilidade de construir uma carreira profissional, ou a possibilidade de ingressar no ensino superior, e ter uma boa carreira profissional fora do âmbito esportivo. O momento dessa decisão torna a segunda opção mais segura e, portanto, mais comum, principalmente para atletas com menor TP. Essa evasão tem como principal motivação a possibilidade de ingressar em uma universidade abrindo mão do esporte competitivo, uma vez que a universidade tem um caráter de formação exclusivamente profissional e de inovação científica e tecnológica, inclusive relacionada ao esporte.

Isto posto, cabe ressaltar as limitações aparentes nesse estudo, como a disposição das respostas em caráter objetivo devido ao instrumento utilizado e a impossibilidade de caracterizar o histórico esportivo dos atletas, o que limita a profundidade das afirmações.

Em síntese, cabe destacar que a produção de conhecimentos científicos relacionados às fases da carreira esportiva pode ser fundamental para que programas de formação esportiva sejam capazes de contemplar aspectos que transcendam as várias facetas do esporte. Nesse sentido, sugere-se estudos sobre a carreira esportiva de universitários de maneira longitudinal, buscando compreender motivações em cada fase, englobando aspectos físicos, psicológicos e sociais.

\section{Conclusão}

A melhora física aparece como motivo mais relevante para atletas do sexo masculino. A relação com o treinador, melhora no condicionamento físico e a persistência em treinar mesmo em condições adversas foram motivos para as atletas mais velhas. Motivos relacionados à estética estiveram associados aos atletas do sexo masculino com maior TP.

Para os motivos de possível abandono do esporte, não realizar os sonhos como atleta foi mais importantes para atletas do sexo masculino, enquanto que o não apoio familiar esteve mais atrelado ao sexo feminino. As atletas mais velhas indicaram que poderiam abandonar se não conseguissem render o esperado e se não estivesse na condição física desejada. Atletas mais jovens de ambos os sexos apontaram que o possível abandono esteve associado a uma relação ruim com o treinador. O menor TP esteve associado à incapacidade de render o desejado por atletas do sexo masculino e a incompatibilidade de estudos e treinos de ambos os sexos. Por fim, atletas de ambos os sexos com menor TP tiveram associação negativa aos motivos atrelados à relação com 0 treinador e aos resultados obtidos.

\section{Referências}

1. Azevedo MF de, Santos WD, Costa FR da, Soares AJG. Formação escolar e formação esportiva: caminhos apresentados pela produção acadêmica. Movimento. 2017;23(1):185-200. 
2. Campos $L T$ da $S$, Vigário $P$ dos $S$, Lürdof SMA. Fatores motivacionais de jovens atletas de vôlei. Rev Bras Ciênc Esporte Impresso. 2011;33(2):30317.

3. Berleze A, Vieira LF, Krebs RJ. Motivos que levam crianças à prática de atividades motoras na escola. J Phys Educ. 2002;13(1):99-107.

4. Weimberg RS, Gould D. Fundamentos da psicologia do esporte e do exercício. $4^{\circ}$ ed. Rio Grande do Sul: Artmed; 2008.

5. Marques MP, Samulski DM. Análise da carreira esportiva de jovens atletas de futebol na transição da fase amadora para a fase profissional: escolaridade, iniciação, contexto sócio-familiar e planejamento da carreira. Rev Bras Educ Física E Esporte. 2009;23(2):103-19.

6. Heinerichs S, Curtis N, Gardiner-Shires A. Perceived levels of frustration during clinical situations in athletic training students. J AthITrain. 2014;49(1):68-74.

7. Carmo JV de M, Mattos F de O, Bara Filho M, Miranda R, Ribas PR, Nunes JL, et al. Validação preliminar de questionário de início, manutenção, mudança e abandono (mimca) no esporte para a língua portuguesa. Conexões. 2008;6 (ed. especial):533-44.

8. Carmo JV de M, Matos F de O, Ribas PR, Miranda R, Filho MB. Motivos de início e abandono da prática esportiva em atletas brasileiros. HU Rev. 2009;35(4):257-64.

9. Afonso GH, Freitas DL, Carmo JM, Lefevre JA, Almeida MJ, Lopes VP, et al. Desempenho motor: um estudo normativo e criterial em crianças da Região Autónoma da Madeira, Portugal. Rev Port Ciênc Desporto. 2009;9(2-3):160-74

10. Lopes $\mathrm{P}$, Nunomura $\mathrm{M}$. Motivação para a prática e permanência na ginástica artística de alto nível. Rev Bras Educ Física E Esporte. 2007;21(3):177-87.

11. Lopes P, Oliveira MS, FáTima CR de, Nunomura M. Motivos de abandono na prática de ginástica artística no contexto extracurricular. Rev Bras Educ Física E Esporte. 2016;30(4):1043-9.

12. Teixeira MDC, Batista MTS, Leite LR, Da Silva SF. Síndrome de burnout em atletas infanto-juvenís e juvenís de voleibol feminino participantes do campeonato brasileiro de seleções. J Phys Educ. 2016;27(e2717).

13. Alfermann D, Stambulova N. Career transitions and career termination. In: Tenenbaum G, Eklund RC, editors. Handbook of Sport Psychology. Hoboken, NJ, USA: John Wiley\& Sons, Inc.; 2012. p. 712-33.

14. Tani G, Meira Junior CM de, Oliveira JA de, Côrrea UC. O dayafter olímpico e a universidade. J Phys Educ. 2009;20(4):485-97.

15. Fontes ACD Vianna RPT. Prevalência e fatores associados ao baixo nível de atividade física entre estudantes universitários de uma universidade pública da região Nordeste - Brasil. Rev Bras Epidemiol. 2009;12(1):20-9.

16. Velásquez MT, Torres-Neira D, Sánchez-Martínez H. Análisis psicológico de la actividad física en estudiantes de una universidad de Bogotá, Colombia. Rev Salud Pública. 2006;8(Sup. 2):1-12.

17. Rodriguez G, Lucas JMA, Alonso JLN. Motivos, Motivacion y Desporte. Salamanca: Tesitex; 1999.

18. Lucas JMA. Análisis de lasmotivaciones de inicio, mantenimiento, cambio y abandono deportivo. Un programa piloto de intervención. [Las Palmas]: Universidade de Las Palmas de Gran Canária; 1998.
19. Molinero O, Salguero A, Alvarez E, Marquez S. Dropout reasons in young spanish athletes: relationship to gender, lype of sport and level of competition. J Sport Behav. 2006;29(3):255-69.

20. Molinero O, Salguero A, Álvarez E, Márquez S. Reasons for dropout in youth soccer: a comparison with other team sports. Eur J Hum Mov. 2010;22:21-30

21. Cervelló EM, Escartí A, Guzmán JF. Youth sport dropout from the achievement goal theory. Psicothema. 2007;19(1):65-71.

22. Fraser-Thomas J, Côté J, Deakin J. Examining adolescent sport dropout and prolonged engagement from a developmental perspective. J Appl and prolonged engagement from

23. Calvo TG, Cervelló E, Jiménez R, Iglesias D, Murcia JAM. Using selfdetermination theory to explain sport persistence and dropout in adolescent athletes. Span J Psychol. 2010;13(02):677-84.

24. Delorme N, Chalabaev A, Raspaud M. Relative age is associated with sport dropout: evidence from youth categories of French basketball: Relative age effect and dropout. Scand J MedSci Sports. 2011;21(1):1208.

25. Interdonato GC, Miarka B, Oliveira AR de, Gorgatti MG. Fatores motivacionais de atletas para a prática esportiva. Motriz. 2008;14(1):63-6.

26. Gilenstam K, Karp S, Henriksson-Larsén K. Gender in ice hockey: women in a male territory: gender in ice hockey. Scand J Med Sci Sports. 2007;18(2):235-49.

27. Hoiness AR, Weathington $B L$, Cotrell AL. Perceptions of female athletes based on observer characteristics. Athletic Insight. 2008;10(1):43-54.

28. Melo GF, Silva AA, Durães G, Cardoso FL, Formiga NS, Sampaio TMV Estereótipos de gênero aplicados a homens atletas praticantes de esportes culturalmente femininos: a percepção de leigos, profissionais da educação física e atletas profissionais. Rev Bras Ciênc E Mov. 2015;23(3):30-7.

29. Mata VA da. Da força física à estética corporal: as mudanças nas relações sociais retratadas no corpo. J Phys Educ. 2008;9(1):35-43.

30. Goll JR, Rachadel-Da-Costa SL, Da-Cruz-Souza MA. Autoestima e autoimagem dos adolescentes praticantes e não praticantes de handebol. Ágora Para Educ Física El Deporte. 2014;16(2):170-87.

31. Brandão MRF, Akel MC. Causas e conseqüências da transição de carreira esportiva: uma revisão de literatura. Rev Bras Ciênc E Mov. 2000;8(1):4958.

32. Barros KS de. Recortes da transição na carreira esportiva. Rev Bras Psicol Esporte. 2008;2(1):01-27.

33. Carlin M, Salguero A, Rosa SM. Análisis de los motivos de retirada de la práctica deportiva y su relación con la orientación motivacional en deportistas universitarios. Cuad Psicol Deporte. 2009;9(1):85-99.

34. Peres L, Lovisolo H. Formação esportiva: teoria e visões do atleta de elite no brasil. J Phys Educ. 2006;17(2):211-8.

35. Vieira LF, Junior JRA do N, Vieira JLL. O estado da arte da pesquisa em Psicologia do Esporte no Brasil. Rev Psicol Deporte. 2013;22(2):501-7. 